\title{
IX.
}

\section{Totalluxation einer Beckenhälfte $\left.{ }^{1}\right)_{x}$ \\ Von}

Dr. J. Riedinger.

Mit 1 Abbildung im Text.

Malgaigne unterschied seiner Zeit (1855) am Becken sechs Arten von Luxationen, nämlich diejenigen der Schambeinsymphyse, der Symphysis sacroiliaca, dieser beiden Symphysen oder des Darmbeines, der beiden Symphyses sacro-iliacae oder des Kreuzbeines, der drei Symphysen zusammen, schliesslich des Steissbeines. Von jeder Art konnte Malgaigne nur eine geringe Anzahl von Fällen anführen und auch seither ist die Kasuistik der Beckenluxationen eine sehr spärliche geblieben. Da zudem die meisten Fälle der älteren Literatur angehören, so könnte man fast behaupten, als isolierte Verletzungen sind die Luxationen des Beckens nur dem Namen nach bekannt. Die relativ häufige Trennung der Ileosakralgelenke pflegt man als Begleiterscheinung von Frakturen aufzufassen. Auch ist die Diagnose einer unkombinierten Beckenluxation am Lebenden nicht leicht. Die einfache Trennung in der Schambeinfuge, die den Gynäkologen besser bekannt ist, wird man nicht zur Luxation rechnen können, da wir unter Luxation nicht eine Diastase, sondern eine seitliche Verschiebung der Gelenkenden verstehen.

Über den ersten mit Hilfe von Röntgenstrahlen diagnostizierten Fall berichtet Linser aus der Tübinger chirurgischen Klinik. Linser erkennt nur noch zwei Arten von Luxationen des Beckens an, nämlich die Luxation der einen Beckenhälfte mit Lösung der Symphysis pubis und sacro-iliaca und die Verrenkungen des Kreuzbeines mit oder ohne Trennung der Schambeinfuge. Von beiden Arten existieren nur je 15 Beobachtungen.

Ein Fall, den man als Totalluxation einer Beckenhälfte beżeichnen kann, d. h. eine derartige Verschiebung, dass beide Hälften des Beckens vollständig ausser Kontakt geraten sind, existiert bis jetzt meines Wissens in

1) Vorgetragen in der physikalisch-medizinischen Gesellschaft zu Würzburg. 
der Literatur nicht. In allen publizierien Fällen handelt es sich nur um partielle Verschiebungen.

Die Beichnung "Totalluxation" hat bekanntlich Kocher für jene Fälle von Luxationen der Wirbelsäule eingeführt, in denen es zu einer vollständigen Trennung und Verschiebung zweier Wirbel gekommen ist. Die gleiche Bezeichnung ist gewiss auch anwendbar für folgenden Fall einer schweren, komplizierten Beckenverletzung, der noch dadurch an Bedeutung gewinnt, dass er am Lebenden, allerdings an einem moribunden Kind, beobachtet wurde. Man könnte hier auch von einer Eventration einer Beckenhälfte reden.

Ein zwei Jahre altes Kind (Knabe) lief in einen langsam im Gang befindlichen Möbelwagen. Es kam von der rechten Seite der Strasse her und geriet unter den zwischen den vier Rädern an Ketten befestigten, mit seinem unteren Teil etwa $1 / 2$ Meter vom Boden entfernten, flach nach unten konvexen, muldenförmigen Anhängeteil. So wurde das Kind, ohne dass jemand einen Laut von ihm hörte, eingeklemmt und überfahren. Die Räder waren jedoch über dasselbe nicht hinweggegangen. Als eine blutige Masse wurde das Kind auf der Strasse aufgelesen und in ein Haus getragen. Das Strassenpflaster vom Ort der Unglücksstelle bis zum Haus zeigte die Spuren stärkerer Blutung. Im Haus selbst ist eine solche nicht mehr eingetreten.

Die Untersuchung des Kindes meinerseits $1 / 2$ Stunde nach der Verletzung orgab folgendes:

Die ganze rechte Beckenhälfte liegt eventriert und ror den zerrissenen Kleidern vor. Nirgends findet sich eine Blutung aus einem Gefüss oder eine nennenswerte parenchymatöse Blutung. Das Kind selbst macht bei Berührungen Abwehrbewegungen und weint. In der Ruhe schliesst es die Augen und schläft. Zuweilen erhebt es aber auch den Kopf und schaut umher, als ob es jemand suche. Die Pupillen sind erweitert. Die Atmung ist abwechselnd bald lebhaft und frequent, bald verlangsamt und oberflächlich. Der Puls ist schwach, fadenförmig, kaum zu zählen. Die Haut ist blass, kalt.

Nach Entfernung der blutig durchdrängten Kleider ist die Verletzung in ihrer ganzen Schwere zu erkennen. Man sieht deutlich an der eventrierten Beckenhälfte die Facies symphyseos, die Spina anterior superior, die Facies auricularis ossis ilium, das Tuber ischiadicum etc. Die Beckenhälfte lässt sich leicht reponieren. Das. Kreuzbein ist an normaler Stelle und nicht abnorm beweglich. Knochenbrüche sind bestimmt nirgends vorhanden.

Das Abdomen und die Peritonealhöhle sind in der inneren Hälfte der Leistengegend geöffnet. Der Darm ist beim Abheben der eingesunkenen Haut in der Tiefe sichtbar. Er ist unverletzt und prolabiert nicht, Bewegungen desselben sind nicht $z u$ erkennen. Die Blase scheint ebenfalls nicht verletzt zu sein.

Die Haut der Leistengegend ist fast bis zum äussersten Ende derselben eingerissen und zerfetzt. Die Muskulatur der Bauchwand ist nicht zerrissen. Die Risswunde erstreckt sich in ihrer grössten Ausdehnung aber nicht nach der Leiste hin, sondern nach dem Oberschenkel hin (cf. Abbildung). Links beginnt sie in der Gegend der Trennungsstelle an der Symphyse (auf der Ab- 
bildung etwas weiter nach links wegen der Abduktionsstellung des linken Beines), dann erstreckt sie sich durch das Perineum und von da über den oberen inneren Abschnitt des Oberschenkels, etwa in der Mitte des letzteren endigend. In der Gegend der Symphyse ist die Haut zipfelartig abgerissen und retrahiert. Das untere Ende der Risswunde am Oberschenkel stellt sich als dreieckigen Schlitz dar. Lateralwärts von dieser Stelle bildet die Haut einen ebenfalls dreieckigen Zipfel, von einem kleineren Querriss am Oberschenkel herrührend. Von Skrotum und Haut des Penis ist nichts zu sehen.

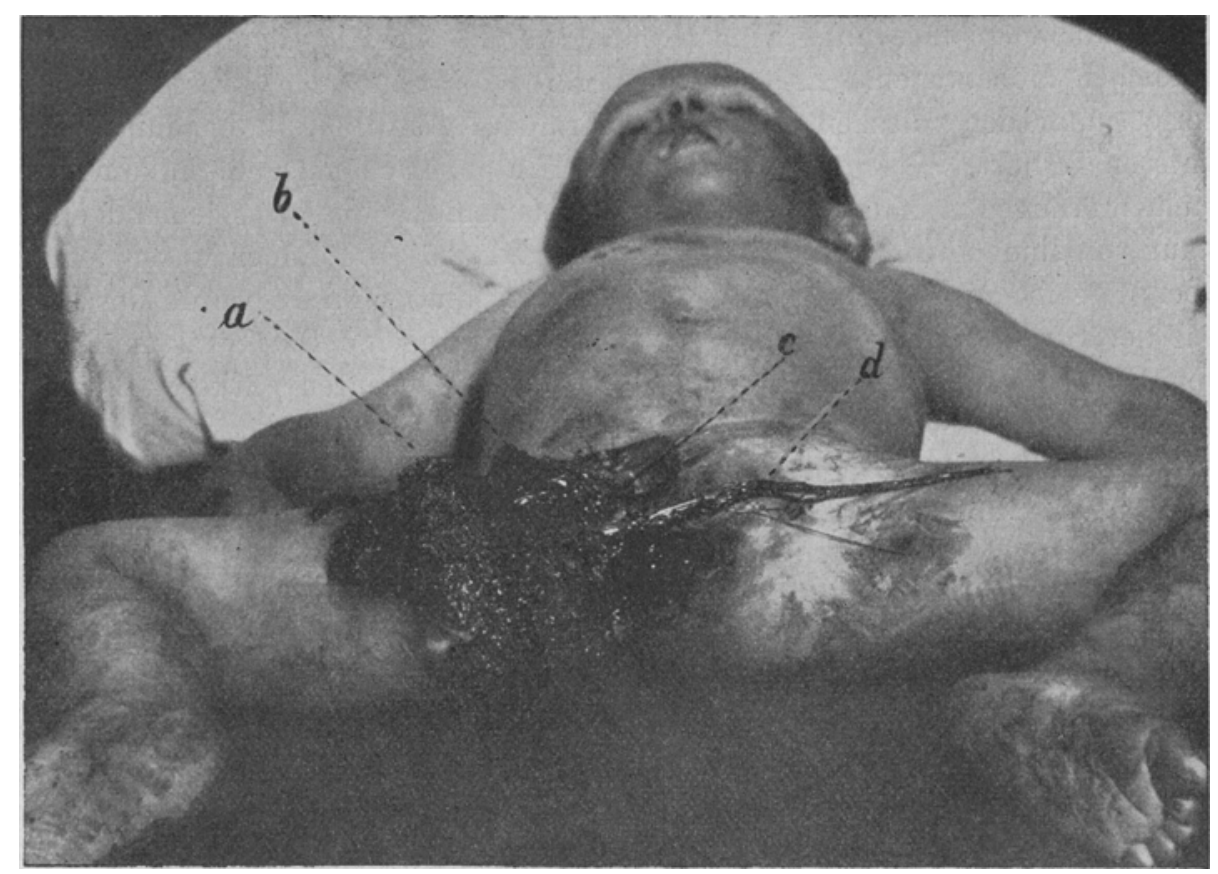

Fig. 1.
$a=$ Facies symphyseos.
$c=$ Facies auricularis o. il.
$b=$ Spina ant. sup.
$d=$ Nervenstämme.

Die Analöffnung ist in ihrem ganzen Umfange erhalten. Die Adduktoren haften am Becken.

Zerrissen sind ausser der Haut die Arteria femoralis, die Vena femoralis und der Nervus cruralis, ferner der Samenstrang und die Harnröhre.

Der Nervus cruralis bildet mit den starken Nervenstämmen des Plexus sacralis einen längeren, aus einzelnen Fäden bestehenden Strang. Die Nerven bildeten, wie es scheint, den resistentesten Teil und sind erst an ibrem Ursprung am Ruickenmark abgerissen. Fin Photogramm, von einer nach dem Ableben des Kindes unter Benützung künstlicher Beleuchtung gemachten Aufnahme herstammend, lässt den rückenmarkartigen, jedoch nicht kompakten Strang deutlich erkennen. 
Dass schliesslich auch der Psoas major und Psoas minor zerrissen waren, lässt sich denken.

Sonstige Verletzungen liegen nicht vor, abgesehen von einigen leichten Abschürfungen im Gesicht und vorn auf der Brust.

Nach dem ganzen Befund war anzunehmen, dass das Becken infolge übermässiger Abduktion und Extension des Oberschenkels zuerst seinen Weg durch das Perineum gesucht hat. Von hier aus erweiterte sich alsdann die durch die Zerreissung gesetzte, grosse Wundfäche nach rechts hin hauptsächlich über den Oberschenkel und über die Gefässe der Leistenbeuge hinaus. Das Kind lebte nach der damahigen Berechnung 21/4 Stunden. Dass nicht sofortige Verblutung eintrat, liegt wohl daran, dass keine glatte Durchtrennung der Gefässe stattfand.

Der Fall ist interessant wegen der Totalität und des Mechanismus der Verrenkung, der Art der Verletzung der einzelnen Teile, sowie der Beobachtung am Lebenden.

Die nächste Therapie hatte sich selbstverständlich nach der Indicatio vitalis zu richten. 